\title{
Glacier Displacement Field Estimation Using Airborne SAR Interferometry
}

\author{
Pau Prats, Christian Andres, Rolf Scheiber, \\ Karlus A. Câmara de Macedo and Jens Fischer \\ German Aerospace Center (DLR) \\ Microwaves and Radar Institute \\ P.O. Box 1116, D-82234, Wessling, Germany \\ Email: Pau.Prats@dlr.de
}

\author{
Andreas Reigber \\ Berlin University of Technology \\ Computer Vision and Remote Sensing Dept. \\ Berlin D-10587, Germany \\ Email: anderl@cs.tu-berlin.de
}

\begin{abstract}
This paper deals with the methodology in the processing of airborne SAR data to measure glacier displacement fields. The possibility to retrieve a $2 \mathrm{D}$ displacement map of the deformation in slant-range geometry with an airborne platform is discussed. A new extended multisquint approach is proposed to simultaneously estimate residual motion errors and the along-track displacement of the glacier, while the across-track displacement is obtained by means of differential interferomatry. Experimental results are shown with data acquired by the Experimental SAR (E-SAR) of the German Aerospace Center over the Aletsch glacier in the Swiss Alps.
\end{abstract}

\section{INTRODUCTION}

Differential SAR interferometry (DInSAR) has already proven its wide range of applications: modelling surface deformation, landslides, soil compaction rate, atmosphere estimation and glacier monitoring, among others. All these applications have been obtained mainly in the spaceborne case thanks to the ideal trajectory of the platform. However, little results have been obtained up to date concerning airborne differential interferometry. The fact that the platform does not follow an ideally linear trajectory does not turn out to be a problem itself, since efficient motion compensation (MoCo) algorithms exist nowadays capable of overcoming this limitation. However, the main drawback is the existence of the so-called residual motion errors (RME): inaccuracies in the order of a few centimeters in the navigation data. Such errors can strongly limit the accuracy of the obtained interferograms, masking completely the deformation movement. Note for example that a $5 \mathrm{~cm}$ error are $156^{\circ}$ at L-band.

Among existing algorithms to estimate RME lie those that estimate the difference between individual RME in an interferogram, i.e. the baseline error [1]. These approaches consist in estimating the azimuth coregistration error between different looks, since such offsets occur due to the presence of RME. The baseline error is retrieved after a proper scaling and integration along azimuth. In the particular approach presented in [1], several looks are combined to extend the coherent areas and reduce phase noise, hence the name of multisquint. Furthermore, a model-based integration based on a least-squares (LS) estimation is also proposed to retrieve the individual vertical and horizontal components of the baseline error. The better the interferometric coherence, the better the performance of multisquint. The main assumption of the multisquint approach, valid in most scenarios, is that any azimuth coregistration offset comes from RME. Therefore, if the scene is experiencing some along-track deformation, a biased estimation of the baseline error will result.

This paper focuses on the modifications applied in the multisquint case in order to avoid a bias in the baseline error estimation due to an along-track movement of the scene, and simultaneously estimate the along-track component of the deformation. Section II analyzes this possibility, while Section III focuses on the particular case of a glacier, where the steps to obtain the 2D displacement field of a glacier by means of differential interferometry (across-track displacement) and spectral-diversity [2] (along-track displacement) are expounded. Finally, Section IV presents first deformation results of the Aletsch glacier, located in the Swiss Alps, with data acquired by the Experimental SAR (E-SAR) of the German Aerospace Center (DLR).

\section{EXTEndED MultisQuint}

When applying multisquint to an interferometric pair, it is assumed that any azimuth coregistration error comes from the difference in the individual RME. In practice, the reference tracks are computed to be parallel and with the same forward velocity in order to obtain the images already aligned along-track after focusing. Therefore, any coregistration error occurring in azimuth is assumed to araise due to RME. This assumption will not be valid whenever an along-track deformation takes places in the scene under observation. This deformation results in a true coregistration error (TCE) that will bias the estimation of the baseline error if not considered. In the following, an approach based on multisquint is proposed to separate these two contributions.

In multisquint [1], $N$ adjacent looks are combined to form $N-1$ spectral diversity phases. These spectral diversity phases contain information about the baseline error, and in particular about the derivative of the baseline error. In order to reduce noise and extend the coherent areas, these spectral diversity phases are shifted to the same beam-center geometry (track geometry) and added in the complex domain. The shift is necessary since the image is focused in a zero-Doppler 
geometry, but the location of the baseline error depends on the aspect angle used to focus the look. Therefore, images need to be aligned to the geometry of the track, i.e. the beam-center geometry. The relation between both geometries is given by

$$
x_{\mathrm{c}}=x_{0}-r_{0} \tan \beta_{i},
$$

where $x_{\mathrm{c}}$ is the beam-center position in meters, $x_{0}$ is the zeroDoppler position, $r_{0}$ is the slant-range distance, and $\beta_{i}$ is the squint corresponding to the center frequency of look $i$. If a TCE is present, its location in the image will not change from look to look unlike the baseline error, hence all spectral diversity phases will have the same information at the same location (zero-Doppler) concerning TCE.

Let $\dot{f}_{\text {be }}(x)$ be the contribution in the spectral diversity phase due to residual motion errors, i.e. the derivative of the baseline error, and $g_{\text {tce }}(x)$ the contribution due to the true coregistration error, where in both cases the range dependence has been omitted for simplicity. For each spectral diversity phase the retrieved signal in zero-Doppler geometry is

$$
s_{i}(x)=\dot{f}_{\text {be }}\left(x-r_{0} \tan \beta_{i}\right)+g_{\text {tce }}(x), \quad \forall i=1, \ldots, N-1 .
$$

By inspecting (2), a first possibility to separate both contributions would be to just add all $s_{i}(x)$ in the complex domain without aligning them to the beam-center geometry, i.e.

$$
\hat{g}_{\text {tce }}(x)=\arg \left\{\sum_{i=1}^{N-1} \exp \left(\mathrm{j} s_{i}(x)\right)\right\} .
$$

In this way, only the contribution due to TCE would remain. However, in order to fairly cancel the contribution of $\dot{f}_{\mathrm{be}}(x)$, the used azimuth bandwidth must be quite large. In practice, the whole $400 \mathrm{~Hz}$ bandwidth of the E-SAR system at Lband, splitted in looks of $60 \mathrm{~Hz}$ and separated $30 \mathrm{~Hz}$, was used. A couple of iterations were needed in this case to achieve a satisfactory performance. Also, the avaiability of more images, either acquired the first or second day, will result in more estimates of $\hat{g}_{\text {tce }}(x)$. Since the baseline error will be decorrelated between interferograms, adding these solutions will improve the estimation. Nevertheless, it should be noted that (3) is basically a mean estimator, so that in the case the baseline errors have low frequency components, a coupling between RME and TCE will arise.

A second approach is proposed that overcomes the limitations of the first one. Starting also from (2), it can be noted how the simple difference between two adjacent spectral diversity phases will completely remove $g_{\text {tce }}(x)$, as it is constant among the spectral diversity phases. Therefore

$$
\begin{gathered}
\dot{s}_{i}(x)=\dot{f}_{\text {be }}\left(x-r_{0} \tan \beta_{i+1}\right)-\dot{f}_{\text {be }}\left(x-r_{0} \tan \beta_{i}\right), \\
\forall i=1, \ldots, N-2 .
\end{gathered}
$$

Moreover, if the center squints are selected to be equispaced, it turns out that (4) is nothing but the second derivative of the baseline error, i.e.

$$
\dot{s}_{i}(x) \approx \ddot{f}_{\mathrm{be}}\left(x-r_{0} \tan \bar{\beta}_{i}\right) . \quad \forall i=1, \ldots, N-2,
$$

where $\bar{\beta}_{i}$ is the mean squint between squints $\beta_{i+1}$ and $\beta_{i}$. Similarly as with multisquint, all differential spectral diversity phases need to be aligned to the same geometry, where now the shift is given by the mean angle $\bar{\beta}_{i}$. After shifting and adding in the complex domain all differential spectral diversity phases, a model-based integration based on a LS estimation as presented in [1] can be carried out to retrieve the second derivative of the horizontal $\ddot{\epsilon}_{\mathrm{y}}$ and vertical $\ddot{\epsilon}_{\mathrm{z}}$ baseline error. Once these two components are estimated, a two step integration is necessary in order to retrieve the baseline error

$$
\epsilon_{m}=\int_{0}^{x_{1}^{\prime}} \int_{0}^{x_{2}^{\prime}} \frac{\ddot{\epsilon}_{m}}{\Delta x_{1} \cdot \Delta x_{2}} d x_{1}^{\prime} d x_{2}^{\prime}, \quad m=\{\mathrm{y}, \mathrm{z}\},
$$

with

$$
\begin{aligned}
\Delta x_{1} & =r_{0} \cdot\left(\tan \beta_{1}-\tan \beta_{0}\right) \\
\Delta x_{2} & =\frac{r_{0} \cdot\left(\tan \bar{\beta}_{1}-\tan \bar{\beta}_{0}\right)}{v} \cdot P R F
\end{aligned}
$$

$\Delta x_{1}$ is the distance in meters between two consecutive looks, while $\Delta x_{2}$ is the distance in pixels between two spectral diversity phases.

The main drawback of this approach is that, besides constant and linear components of the baseline error, also the quadratic component of the baseline error remains unknown. The original multisquint approach has the main limitation that constant and linear terms cannot be estimated. In [1] a solution is proposed to estimate them by means of an external DEM and a LS estimation. Similarly, the quadratic component can be added to the model to estimate it. The procedure consists in subtracting to the interferometric phase the synthetic phase computed with the DEM. Afterwards, the phase is unwrapped and by means of a LS estimation constant, linear and quadratic terms are obtained. However, it should be noted that the line of sight (LOS) displacement in the scene can introduce a bias in these components. Therefore, any area experiencing some deformation should be masked. In the presented results the location of the glacier is well known, so that a mask can be easily generated.

Another minor drawback is the fact that a two step integration must be carried out. The performance will mainly depend on the coherence of the scene, but in practice several iterations should be carried out to better estimate the baseline error. Once the baseline error is estimated, the track of the slave image can be updated with $\epsilon_{\mathrm{y}}$ and $\epsilon_{\mathrm{z}}$, so that after re-processing both images will have the same RME, hence cancelling out after interfereogram generation. Finally, any azimuth coregistration error in the scene will correspond to a true coregistration error, i.e. a displacement in the scene. An efficient approach to estimate these displacements is presented in [2] and it has been used in the results presented in Section IV.

The main assumption made in the extended multisquint approach is that the azimuthal coregistration error is not larger than the pixel resolution of a look. This usually applies for residual motion errors with the E-SAR system. However, this might not be the case with the azimuthal glacier movement, so that special care must be taken in this case. Nevertheless, 


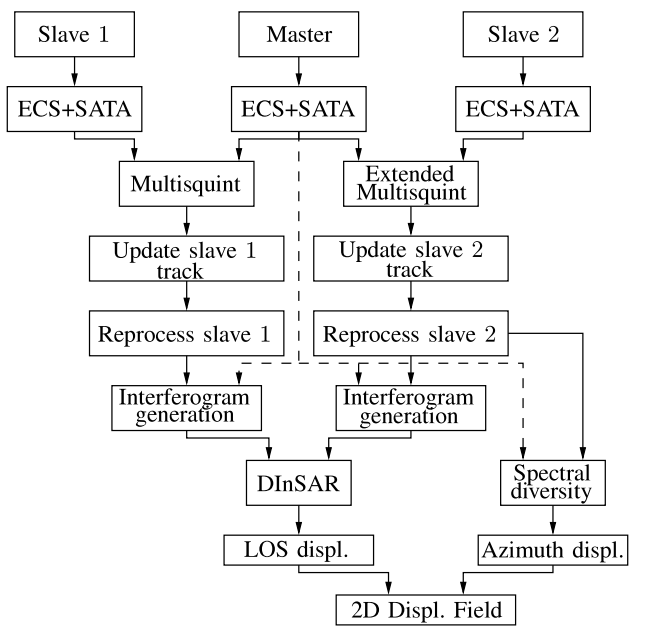

Fig. 1. Block diagram of the implemented processing chain to obtain the 2D displacement field of a glacier

in the results shown in Section IV the maximum azimuthal displacement of the glacier is around half of the azimuthal image resolution of the SLC for a processed bandwidth of $100 \mathrm{~Hz}$

It is worth mentioning a third possibility to separate RME and TCE: to align all spectral diversity phases to the beamcenter geometry and then make the difference between consecutive looks instead of adding them. In this way, the contribution due to RME is cancelled, and only the derivative of TCE remains. The differential spectral diversity phase must be integrated along the azimuthal direction to retrieve TCE. This integration step is more critical than before as a $2 \mathrm{D}$ matrix has to be integrated instead of a vector. Currently some approaches are under study towards this third possibility.

\section{2D Displacement FiELd Estimation}

In the previous section it has been shown how to separate RME and TCE in an interferogram where along-track displacements due to surface movement are occurring. This Section expounds the processing chain in order to retrieve the 2D displacement field of a glacier using three images, with two of them acquired the same day, and the third one acquired after a certain time baseline. Fig. 1 shows the block diagram of the implemented processing chain. Master image and slave image 1 are assumed to be acquired the same day in a very short time span (about 15 minutes in the presented results), while slave image 2 is acquired after some displacement in the scene has occurred (after 1 day in the presented results).

The processing starts with the focusing of the images. In the implemented processing chain, the Extended Chirp Scaling (ECS) algorithm [3] has been used. Since there is strong topography in the scene, a topography-dependent algorithm is needed to avoid the coupling between residual motion errors and DEM errors, since an unknown height can result in a displacement of the impulse response, and consequently, in a coregistration error. In the presented results, the Sub-Aperture Topography- and Aperture dependent (SATA) algorithm was used, but any other algorithm is also suitable [4]. After the focusing, multisquint and extended multisquint (averaging approach or two-step integration approach) are applied to the corresponding image pairs, i.e. master-slave 1, and masterslave 2, respectively. The external DEM is also used here to estimate constant and linear terms of the baseline error for the short-term interferogram, and including the quadratic term for the long-term interferogram provided that the twostep integration approach is used. Once the baseline errors have been estimated, the slave tracks are updated and the slave images reprocessed. The fact that the whole baseline error is applied to the slave images implies that now all the images have the same RME as master. Hence, any generated interferogram will result RME-free. The last step is to estimate the displacements in the scene. For the LOS displacement DInSAR achieves the best possible accuracy, hence a differential interferogram is generated using all three images. To estimate the along-track displacement, any technique to estimate the coregistration offsets is suitable. In the presented case, spectral diversity [2] has been used, as it can achieve very good accuracy [5].

A further step in the case of a glacier is possible. If the surface parallel flow assumption is taken, i.e. no emergence/submergence is considered, and it is also assumed that the glacier flows in the direction of the maximum slope, the 2D displacement field in the slant-range plane can be projected using the external DEM to retrieve the 3D displacement field.

One important drawback worth mentioning is the coupling between RME and unknown topography. A shift of the impulse response will occur whenever the external DEM has errors. The magnitude of this displacement depends also on the magnitude of the track deviations of the platform. Therefore, this undesired coregistration offset can bias the estimated baseline error when using multisquint. In the three tracks involved in the results shown in next section, horizontal and vertical deviations are within $\pm 4 \mathrm{~m}$. Assuming a $2 \mathrm{~m}$ error in the X-band DEM used for motion compensation, the induced bias in the baseline error for a target at mid range is within $\pm 2 \mathrm{~mm}$, which gives a hint on the lower bound accuracy of the estimated LOS results.

\section{EXPERIMENTAL RESULTS}

In order to validate the presented approach, results with data acquired by the E-SAR system of DLR over the Aletsch glacier in the Swiss Alps are shown. Data were acquired the $27^{\text {th }}$ and $28^{\text {th }}$ of October 2003 at L-band, and have been processed with a $100 \mathrm{~Hz}$ azimuth bandwidth and a $100 \mathrm{MHz}$ range bandwidth, while the platform speed is $95.1 \mathrm{~m} / \mathrm{s}$. The shortterm interferogram was acquired with a $10 \mathrm{~m}$ baseline, while the long-term has a zero-baseline acquisition configuration. Fig. 2(a) shows the reflectivity image of the scene and Fig. 2(b) the external DEM obtained from the X-band data in singlepass mode acquired the second day. As it can be noted, the strong topography variations require a topography-dependent algorithm to properly focus the data and avoid image degradation. After following the processing chain depicted in Fig. 1, 

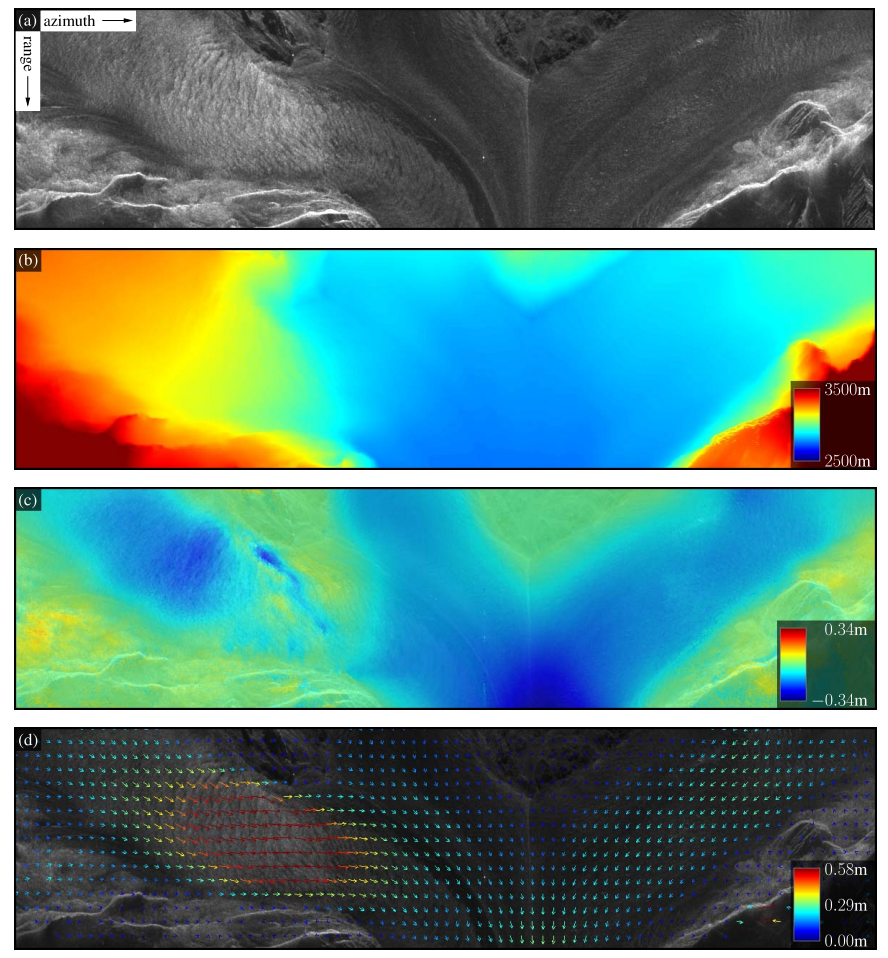

Fig. 2. (a) Reflectivity image of the Aletsch glacier, (b) X-band DEM used during motion compensation, (c) one-day differential interferogram with overlayed reflectivity, and (d) one-day estimated 2D displacement field with overlayed reflectivity. Scene dimensions: $6.8 \mathrm{~km} \times 1.4 \mathrm{~km}$.

which includes the proposed extended multisquint, the alongtrack displacement between master image and the long-term slave image is estimated using spectral diversity. Similarly, spectral diversity can also be used to estimate the acrosstrack misregistration. With this information, the long-term slave image can be interpolated to improve the quality of the interferogram. Afterwards, the LOS displacement is obtained by means of differential interferometry using three images. The result is depicted in Fig. 2(c), where the movement of the glacier can be nicely observed. It should be noted that the accuracy in the estimation of the along-track displacement is not as good as with the LOS displacement, but since the longterm coherence is quite good (mean of 0.78 ), very accurate estimates can be obtained. Finally, the 2D displacement field in slant-range geometry depicted in Fig. 2(d) is obtained. The only in-situ measurement available corresponds to a corner reflector, whose position was measured the $22^{\text {nd }}$ of October and the $5^{\text {th }}$ of November, yielding a mean displacement in LOS and azimuth of $13.6 \mathrm{~cm} /$ day and $10.1 \mathrm{~cm} /$ day, respectively. The estimated deformation is $15.2 \mathrm{~cm} /$ day in $L O S$ and $9.6 \mathrm{~cm} /$ day in azimuth after applying the proposed techniques. Fig. 3 shows a zoom on the corner reflector. Note that no corner reflectors were used to calibrate the data, but only the external DEM.

\section{CONCLUSION}

This paper has shown for the first time the possibility to retrieve the 2D displacement field of a glacier using airborne SAR interferometry. The main drawback to accurately process

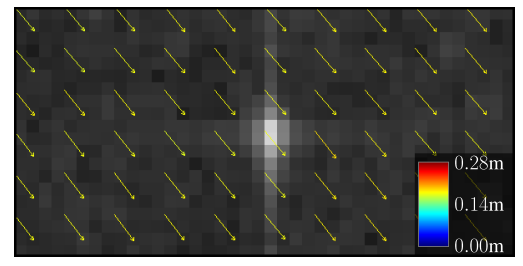

Fig. 3. Zoom showing the estimated displacement of the glacier around the corner reflector.

airborne interferometric data is the presence of RME. Usually, interferometric techniques based on the azimuth coregistration offsets between looks are used to estimate the baseline error. Therefore, any movement of the scene itself in the alongtrack direction can lead to a biased estimation of the baseline error, and hence, to a degraded result. In this paper, a solution has been proposed to overcome this limitation, which makes use of the different nature of RME and TCE. Exemplary results have been shown with data acquired over the Aletsch glacier with the E-SAR system of DLR, demostrating the potential of airborne platforms to accurately monitor deformation phenomena. Furthermore, airborne systems offer an extended flexibility in the data acquisition configuration, as well as the possibility to use different frequency bands. Future work will address other possibilities to decouple RME from TCE avoiding the double integration, as well as alternative approaches like the use of autofocus techniques using isolated or point-like scatterers [6], [7].

\section{ACKNOWLEDGMENTS}

The authors would like to thank the RSL team of University of Zürich for campaign preparation and ground measurements and the E-SAR team of DLR for the flights. The SWISAR campaign was performed with funding of "armasuisse" Switzerland.

\section{REFERENCES}

[1] A. Reigber, P. Prats, and J. J. Mallorqui, "Refined estimation of timevarying baseline errors in airborne SAR interferometry," IEEE Geosci. Remote Sensing Lett., vol. 3, no. 1, pp. 145-149, Jan. 2006.

[2] R. Scheiber and A. Moreira, "Coregistration of interferometric SAR images using spectral diversity," IEEE Trans. Geosci. Remote Sensing, vol. 38, no. 5, pp. 2179-2191, July 2000.

[3] A. Moreira, J. Mittermayer, and R. Scheiber, "Extended chirp scaling algorithm for air- and spaceborne SAR data processing in stripmap and scanSAR imaging modes," IEEE Trans. Geosci. Remote Sensing, vol. 34, no. 5, pp. 1123-1136, Sept. 1996.

[4] P. Prats, K. A. C. de Macedo, A. Reigber, R. Scheiber, and J. J. Mallorqui, "Comparison of topography- and aperture dependent motion compensation algorithms for airborne SAR," IEEE Geosci. Remote Sensing Lett., vol. 4, no. 3, July 2007.

[5] R. Bamler and M. Eineder, "Accuracy of differential shift estimation by correlation and split-bandwidth interferometry for wideband and Deltak SAR systems," IEEE Geosci. Remote Sensing Lett., vol. 2, no. 2, pp. 151-155, Apr. 2005.

[6] H. Cantalloube and P. Dubois-Fernandez, "Airborne X-band SAR imaging with $10 \mathrm{~cm}$ resolution: technical challenge and preliminary results," IEE Proc.-Radar Sonar Navig., vol. 153, no. 2, pp. 163-176, Apr. 2006.

[7] K. A. C. de Macedo, R. Scheiber, and A. Moreira, "An autofocus approach for residual motion errors with application to airborne repeat-pass SAR interferometry," in Proc. IEEE IGARSS 2007, Barcelona, Spain, July 23$27,2007$. 